ceased. The IPC-ablated larvae had an average combined glucose and trehalose level of $38 \%$ above normal, and these levels returned to normal when DILP2 was provided systemically by the transgene (Fig. 1C). Elevated hemolymph carbohydrate levels in larvae lacking IPCs indicate that insulin is an essential regulator of energy metabolism in Drosophila. This accumulation of carbohydrate in the blood is reminiscent of that seen in human diabetes mellitus, although it should be noted that we measured carbohydrate levels during development rather than in adults.

To investigate how central nervous system (CNS)-derived insulin regulates systemic functions, we examined the Drosophila IPC contacts outside the CNS. The morphology of the brain IPCs was examined with the use of the dilp2 promoter to drive expression of mGFP, a membrane bound green fluorescent protein (GFP) (Fig. 2, A to C). The IPC clusters within the pars intercerebralis extend processes that terminate at the lateral protocerebrum and subesophageal ganglion. IPC processes also terminate on the heart and in the corpora cardiaca (CC) compartment of the ring gland, after crossing the midline and exiting the CNS (Fig. 2F). Labeling of the IPC processes with mGFP and DILP2 antibody (fig. S1) revealed localization of DILP2 peptide within the processes that contact the heart and ring gland (Fig. 2A). DILP2 peptide was concentrated in a graded distribution outside the cells of synthesis on the heart (Fig. 2C), and colabeling with myosin heavy chain antibody, which labels the columnar heart epithelium, showed that the IPC processes and DILP2 were localized outside the lumen of the heart (Fig. 2D). These results suggest the heart surface may be the site of insulin release to the openly circulating hemolymph. We propose that brain IPCs are essential for organismwide growth control and carbohydrate homeostasis through release of insulin peptides into circulating hemolymph. These cellular functions are notably similar to those of mammalian pancreatic $\beta$ cells $(6,7)$.

In Drosophila (8) and other insects (9), a fraction of CC cells synthesize adipokinetic hormone (AKH). AKH resembles glucagon in its activation of glycogen phosphorylase through heteromeric GTP-binding protein (G protein) and adenosine $3^{\prime}, 5^{\prime}$-monophosphate (cAMP) signaling to elevate blood sugar (10), and the two proteins have some limited sequence similarity (fig. S5). Double labeling of AKH mRNA and DILP2 peptide showed that IPCs extend processes to the $\mathrm{CC}$ and that $\mathrm{AKH}$-expressing cells contain DILP2 (Fig. 2E). We also found that CC cells accumulate DILP2 within membrane-bound particles of the perinuclear space, suggesting the possibility that DILP2 is taken up by AKH cells (fig. S6). We cannot formally rule out the possibility that dilp2 is transiently or minutely transcribed by AKH cells, although we have not detected expression of either the dilp2 promoter or dilp1, $-2,-3$, and -5 mRNAs in the AKH cells. Thus, in addition to contacts between IPCs themselves, the primary sites of IPC contact outside the CNS are the heart and the CC. Though they lack strict morphological homology, these intercellular contacts are analogous to the association of pancreatic $\beta$ cells with other $\beta$ cells, with glucagonexpressing $\alpha$ cells, and with blood vessels in the islets of Langerhans (7) and may reflect underlying evolutionary conservation.

Thus, there is remarkable similarity of the organ systems underlying conserved insulin function in diptera and mammals. Moreover, the presence of IPCs in the nervous systems of other invertebrate and protochordate species ( 1 , 11-14) and in primary cell cultures from mammalian fetal brain (15) provides further evidence for a common ancestral insulin-producing organ of neural origin. Our results also raise the possibility that common mechanisms of cell specification regulate development of pancreatic $\beta$ cells and Drosophila brain IPCs.

\section{References and Notes}

1. W. Brogiolo et al., Curr. Biol. 11, 213 (2001).

2. S. L. McNabb et al., Neuron 19, 813 (1997).

3. C. Chen, J. Jack, R. S. Garofalo, Endocrinology 137, 846 (1996).
4. R. Bohni et al., Cell 97, 865 (1999).

5. G. R. Wyatt, Annu. Rev. Entomol. 6, 75 (1961).

6. B. Duvillie et al., Proc. Natl. Acad. Sci. U.S.A. 94, 5137 (1997).

7. E. P. Joslin, C. R. Kahn, G. C. Weir, Joslin's Diabetes Mellitus (Lea \& Febiger, Philadelphia, PA, ed. 13, 1994).

8. B. E. Noyes, F. N. Katz, M. H. Schaffer, Mol. Cell. Endocrinol. 109, 133 (1995).

9. H. F. Nijhout, Insect Hormones (Princeton Univ. Press, Princeton, NJ, 1994)

10. S. F. Vroemen, D. J. Van der Horst, W. J. Van Marrewijk, Mol. Cell. Endocrinol. 141, 7 (1998).

11. H. Kondo, M. Ino, A. Suzuki, H. Ishizaki, M. Iwami, J. Mol. Biol. 259, 926 (1996).

12. A. B. Smit et al., Nature 331, 535 (1988).

13. S. B. Pierce et al., Genes Dev. 15, 672 (2001).

14. J. E. McRory, N. M. Sherwood, DNA Cell Biol. 16, 939 (1997).

15. D. W. Clarke, L. Mudd, F. T. Boyd Jr., M. Fields, M. K. Raizada, J. Neurochem. 47, 831 (1986).

16. We thank K. Willert for help in antibody production, J. Peterson for help with in situ hybridization, and $M$. Fish for help with $P$ element transformation. Supported by the Juvenile Diabetes Research Foundation (E.J.R.) and the Pew Charitable Trusts (S.K.K.).

Supporting Online Material

(www.sciencemag.org/cgi/content/full/296/5570/1118/ DC1)

Materials and Methods

figs. $\mathrm{S} 1$ to $\mathrm{S} 6$

22 January 2002; accepted 1 April 2002

\title{
Stability in Real Food Webs: Weak Links in Long Loops
}

\author{
Anje-Margriet Neutel, ${ }^{1 *}$ Johan A. P. Heesterbeek, ${ }^{2}$ \\ Peter C. de Ruiter ${ }^{1}$
}

\begin{abstract}
Increasing evidence that the strengths of interactions among populations in biological communities form patterns that are crucial for system stability requires clarification of the precise form of these patterns, how they come about, and why they influence stability. We show that in real food webs, interaction strengths are organized in trophic loops in such a way that long loops contain relatively many weak links. We show and explain mathematically that this patterning enhances stability, because it reduces maximum "loop weight" and thus reduces the amount of intraspecific interaction needed for matrix stability. The patterns are brought about by biomass pyramids, a feature common to most ecosystems. Incorporation of biomass pyramids in 104 foodweb descriptions reveals that the low weight of the long loops stabilizes complex food webs. Loop-weight analysis could be a useful tool for exploring the structure and organization of complex communities.
\end{abstract}

An increasing number of studies provide evidence for the occurrence in real biological communities of patterns of interaction strengths that are important to community stability $(1-8)$. Such patterns are character-

${ }^{1}$ Department of Environmental Sciences, Utrecht University, Post Office Box 80115, 3508 TC Utrecht Netherlands. ${ }^{2}$ Faculty of Veterinary Medicine, Quantitative Veterinary Epidemiology Group, Utrecht University, Post Office Box 80151, 3508 TD Utrecht, Netherlands.

*To whom correspondence should be addressed. Email: a.neutel@geog.uu.nl ized, for example, by strong links embedded in a majority of weak links $(1,2,6)$ or by strong links concentrated on the lower trophic levels (5). However, there remains a need to determine which food-web properties cause the patterns, and to understand the exact form of the patterns and how they influence stability. Here we analyze the stabilizing properties of observed patterns in food webs $(5,9)$ by looking at the distribution of interaction strengths $(10,11)$ over trophic loops $(12-14)$ of different lengths.

We carried out our first analysis with 
Fig. 1. Feeding relations (A) and loops (B) in the CPER food web. Interaction strengths (values in parentheses), shown for the feeding relations in bold arrows, are the values as published for the CPER food web (9). (B) A single predatorprey relation forms a loop of length 2 , containing a bottom-up effect (prey on predator) and a top-down effect (predator on prey) (I, II). The weight of this particular loop in the CPER food web was 0.55 per year (geometric mean of the absolute values of the interaction strengths). (C) Apart from three loops of length 2, a three-species omnivorous relation forms two loops of length 3: a loop containing two top-down effects and one bottom-up effect (I, II, III), and one containing two bottom-up effects and one top-down effect $\left(\mathrm{I}^{\prime}, \mathrm{II}^{\prime}, \mathrm{III}^{\prime}\right)$. The weights of these loops were 0.14 and 0.10 per year, respectively.
A 4

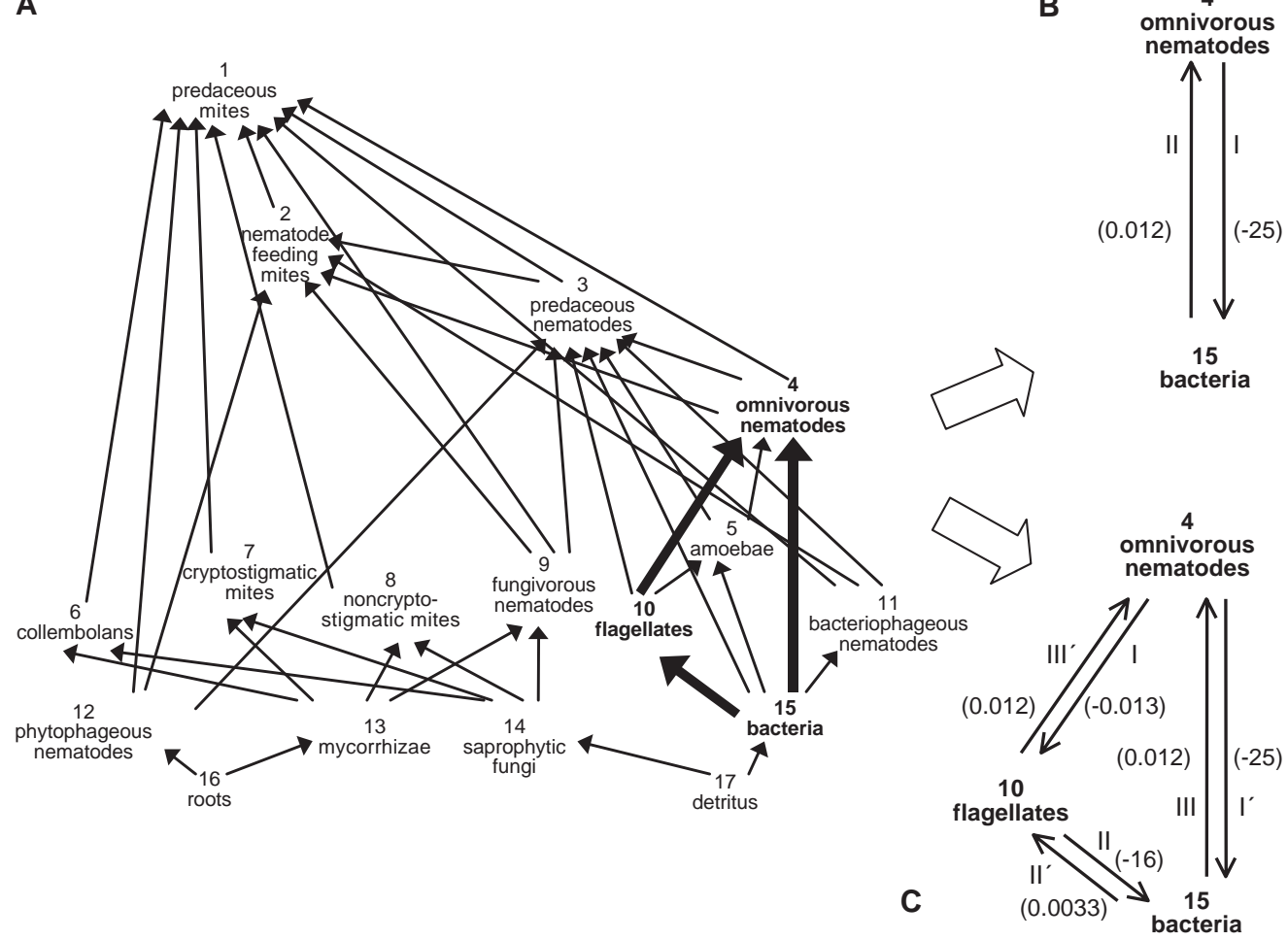

15

bacteria seven documented soil food webs $(15,16)$.

The data collected for these communities enabled us to estimate population sizes, feeding rates, and interaction strengths among species $(5,9,17)$. Throughout the analysis, species refers to trophic species or functional groups in which species are aggregated that share the same prey and predators and have similar physiological characteristics (18). With respect to interaction strengths, we follow May (10); interaction strengths are the values of the elements of the (Jacobian) community matrix and represent the size of the effects of species on each other's dynamics near equilibrium $(10,11)$. We present results obtained from the food web of the central plains experimental range (CPER) as a representative example of the seven food webs.

As indicated in the diagram of the CPER food web (Fig. 1A), a loop describes a pathway of interactions from a certain species through the web back to the same species without visiting other species more than once; hence, a loop is a closed chain of trophic links (14). Each step on the path going from one species to another refers to the interaction between the two species (Fig. 1, B and C), which can be either a positive bottom-up effect or a negative top-down effect. The length of a loop is the number of different species visited. Loops of length 2 thus go from a predator to a prey and back (Fig. 1B). Longer loops are formed wherever food chains separate and are connected again (through either polyphagy or omnivory) (Fig. 1C). A three-species omnivorous relation forms two loops of length 3 , one with two
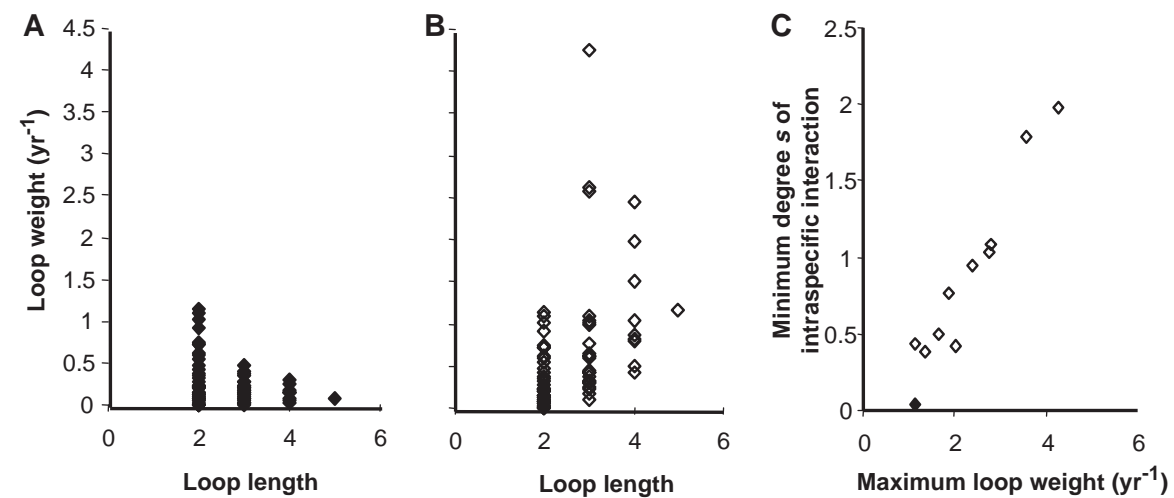

Fig. 2. Loop length, loop weight, and stability in the CPER food web and randomizations (20) of this matrix. (A) Loop weight versus loop length in the real matrix. (B) Loop weight versus loop length in a randomized matrix (a typical example). Long loops with a relatively small weight-those with many bottom-up effects-are not shown because they are not relevant for maximum loop weight. (C) Maximum loop weight and stability of the real matrix (solid diamond) and of 10 randomized matrices (open diamonds). We measured stability as the value $s$ that leads to a minimum level of intraspecific interaction strength needed for matrix stability (11). In a sensitivity analysis, we found that variation in the parameter values within intervals between half and twice the observed value led to only a small variation in stability (22).

top-down effects and one bottom-up effect, and the other with two bottom-up effects and one top-down effect (Fig. 1C).

Originally, a loop is quantified as a product of interaction strengths (14). Here, we introduce the term "loop weight," which is defined as the geometric mean of the absolute values of the interaction strengths in the loop. Quantifying trophic loops in this way allows us to compare loops of different lengths and to use the maximum of all loop weights as an indicator for matrix stability. This indicative value can be understood as follows. Consider community matrix $\mathbf{A}=\left(\alpha_{i j}\right)$, where all elements have been specified except the diagonal elements $\alpha_{i i}, \alpha_{i i}<0$. These diagonal elements refer to the intraspecific interactions. By making the absolute value of $\alpha_{i i}$ large enough, community matrix $\mathbf{A}$ can be stabilized (all its eigenvalues will have negative real parts). We found that the maximum of all the loop weights in community matrix $\mathbf{A}$ is an approximation of the absolute value of $\alpha_{i i}$ sufficient for matrix stability (19).

In the CPER food web, we characterized the observed interaction strengths (9) in terms 

ent lengths, maximum loop weight, and matrix stability. We compared the community matrix, including the patterned interaction strengths ("real matrices"), with a number of randomizations of this matrix ("randomized matrices"). We acquired the randomized matrices by randomly exchanging predator-prey pairs of interaction strengths, keeping these pairs as such intact and preserving the sign structure of the matrix $(5,7,20)$. We measured stability as the minimum degree of relative intraspecific interaction needed for matrix stability $(s)$; we called matrices with a smaller $s$ "more stable" (11).

We found that loop weights of the longer loops were low in the real matrix, whereas in

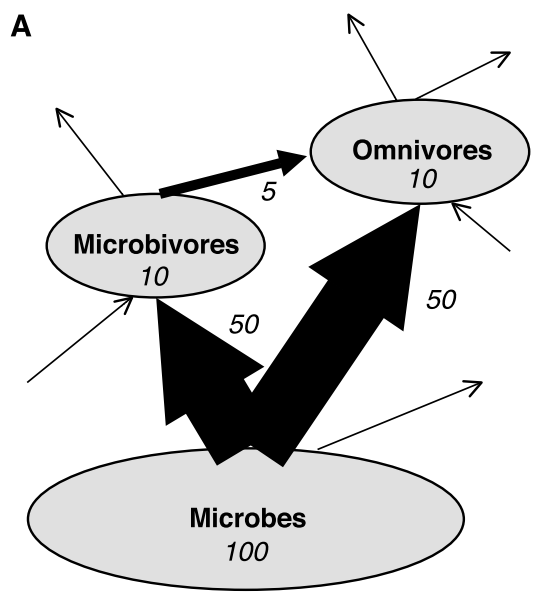

B

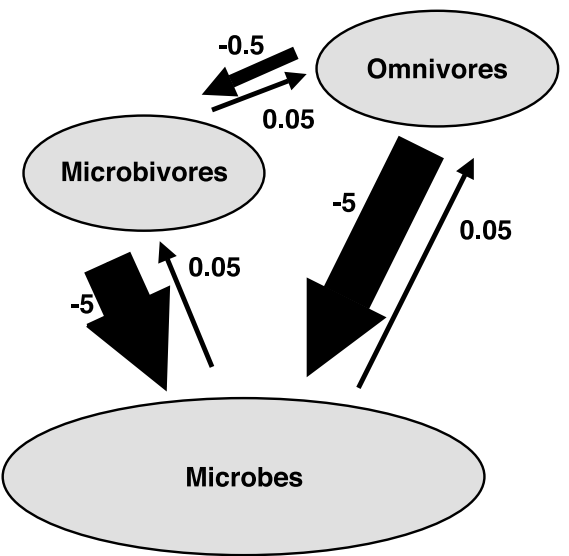

Fig. 3. Interaction strengths and loop weights in an omnivorous food web. (A) Equilibrium feeding rates and population sizes. Feeding rates were assumed to be proportional to the population sizes of the prey. (B) Interaction strengths (11). In the example, we assumed efficiencies of 0.1 for all species. The loop weights of the two loops of length 3 are $(|-0.5| \times|-5| \times 0.05)^{1 / 3}=0.5$ (anticlockwise loop, starting with the omnivores) and $(|-5| \times 0.05 \times 0.05)^{1 / 3}=0.23$ (clockwise loop). The relatively small top-down effect $(-0.5)$ keeps the weight of the loop with two top-down effects relatively low (see text).

randomized matrices the longer loops tended to be heavier than the shorter loops (Fig. 2, A and B). This can be explained as follows. It is a well-known regularity that the absolute values of the effects of predators on their prey are, on average, two orders of magnitude larger than the values of the effects of prey on the CPER $(7,21)$. This was also found in izations of the CPER web. Therefore, loops are expected to be heavy when they contain many top-down effects relative to bottom-up effects. This explains the heavy long loops in matrices. But in the real maeffects had a relatively low weight. This showed that not all top-down or bottom-up of the weights of the various loops of differ-

Fig. 4. Relation among complexity, maximum loop length, maximum loop weight, and stability in a set of 104 food webs (24). Community matrices were derived in the same way as in the analysis of the CPER food web $(5,11)$. We made the simplifying assumption that species feed according to prey abundance and assumed equal conversion efficiencies $e_{i j}=e$ $=0.1$ and equal specific natural death rates $d_{j}=d=-1$ ( per year) for all species. Equilibrium population sizes in each food web were obtained by imposing a simple pyramidal biomass structure with a factor of 20 biomass decrease over trophic levels (22). Each web was represented by 100 pyramidal matrices constructed by drawing matrix elements randomly from uniform intervals $\langle 0$, $\left.2 \alpha_{i j}\right\rangle$ around the derived values of interaction strengths $\alpha_{i j}$. For each pyramidal matrix, a randomized counterpart was derived (20). Maximum loop weight and stability refer to theaverage value of 100 matrices. (A) Maximum loop length versus complexity [the product of the number of species $(n)$ and connectance (C)]. (B) Maximum loop weight versus maximum loop length of the pyramidal matrices. (C) Maximum loop weight versus maximum loop length of the randomized matrices. (D) Stability versus maximum loop length of the pyramidal matrices. (E) Stability versus maximum loop length of the randomized matrices. effects were equal, and it indicated that in the real matrices these long loops contained many relatively small top-down effects. The consequence was that maximum loop weight in the real matrix was much lower than in the randomized matrices; along with this, as expected (19), the real matrix was much more stable (Fig. 2C). Moreover, we found a close correspondence between maximum loop weight and stability within the set of randomized matrices (Fig. 2C). We obtained similar results for the other soil food webs. A sensitivity analysis, evaluating the effects of variation in the input parameter values, showed the general robustness of the stability analysis (22).

A strong biomass decrease over trophic levels, together with the fact that predators may feed on several prey types, caused weak links to aggregate in long loops. The principle is illustrated in Fig. 3 for a threespecies omnivorous relation. The omnivore feeds on two prey types, which are at different trophic levels. We assume that it feeds according to prey abundance, and that the biomass of the prey on the lower trophic level is much larger than that of the prey on the higher trophic level (the biomass pyramid). Then the omnivore feeds largely on the lowest trophic level (23). As a result, it exerts a relatively large top-down effect on its lowest prey and a relatively small topdown effect on its highest prey, because the top-down effect is the feeding rate per unit of predator biomass (11). The long loop with two top-down effects contains this relatively small effect of the omnivore, together with the large top-down effect of the intermediate predator on the bottom prey; that is, the two relatively large top-down effects are in different loops (Fig. 3B). 


\section{R E P O R T S}

Hence, the result of biomass pyramids is that long loops with relatively many topdown effects always contain at least one relatively small top-down effect: the effect of the omnivorous predator on its high trophic-level prey. Randomization (20) means that large top-down effects from different trophic loops may end up in the same loop, causing very high loop weights, leading to instability.

We tested the effect of biomass pyramids on loop weight and stability with the use of 104 food webs in the collection assembled by Cohen et al. (24). We imposed a pyramidal biomass structure on these webs (22) and calculated interaction strengths for all these communities (11), carrying out the same analysis as was done for the soil food webs (Fig. 4). Notwithstanding the acknowledged shortcomings of this particular data set and the criticisms it has attracted $(3,25)$, the analysis reveals that the 104 pyramidal (derived from pyramidal structure) webs had the same characteristic distribution of loop weights over loop lengths as did the CPER web-that is, long loops had low loop weights (as shown in Fig. 2A) - giving these webs a lower maximum loop weight and a much lower level of intraspecific interaction needed for stability than their randomized (20) counterparts. Looking at the complexity of the 104 webs, we found that the more complex webs contained relatively long loops (Fig. 4A) but had roughly the same maximum loop weight and stability as less complex webs with shorter loops (Fig. 4, B and D). In the set of randomized matrices, webs with longer loops had a larger maximum loop weight and were less stable than webs with shorter loops (Fig. 4, C and E). Varying the shape of the trophic pyramid in the range from no decrease to a factor of 50 decrease of biomass per trophic level showed that roughly a factor of 10 biomass decrease over trophic level was sufficient to generate strong stabilizing patterns of interaction strengths. As more extreme decreases in biomass over trophic levels are very common in natural ecosystems (26), the results identify a regularity in a broad class of ecosystems: In complex food webs with pyramids of biomass, long loops contain weak links, resulting in a low maximum loop weight and a low level of intraspecific interaction needed for stability (27).

Stabilizing effects of patterns of interaction strengths have been put forward by empiricists and theoreticians. Establishing interaction strengths in species manipulation and perturbation experiments, Paine (1) and Wootton (2) showed that communities are characterized by a few strong links embedded in a majority of weak links. Yodzis (7) demonstrated the importance of the patterning of strong and weak links for model stability. De
Ruiter et al. (5) showed the importance of energetic organization for food-web stability. McCann et al. (6) revealed that particular combinations of weak and strong links are crucial to stability. This analysis contributes to these findings by showing that stabilizing combinations of strong and weak links are organized in trophic loops, linking this organization to trophic pyramids and explaining the effect in terms of general mathematical stability criteria. The potentially destabilizing effect of long loops is well known (12-14, 28 ); our results indicate how weak links prevent complex food webs with long loops from being unstable (22). Although it remains difficult to decide on the level of detail with which feeding relations are distinguished, loop-weight analysis could be a useful tool for assessing the structure and organization of complex networks of ecological relations. Characterizing complex structures is relevant, because it is often on this level of multitrophic communities that questions about the fragility of system structure and functioning are most pressing.

\section{References and Notes}

1. R. T. Paine, Nature 355, 73 (1992).

2. J. T. Wootton, Ecology 75, 151 (1994).

3. G. A. Polis, Aust. J. Ecol. 19, 121 (1994).

4. B. A Menge, E. L. Berlow, C. A. Blanchette, S. A. Navarrete, S. B. Yamada, Ecol. Monogr. 64, 249 (1994).

5. P. C. De Ruiter, A. M. Neutel, J. C. Moore, Science 269, 1257 (1995).

6. K. McCann, A. Hastings, G. R. Huxel, Nature 395, 794 (1998).

7. P. Yodzis, Nature 289, 674 (1981).

8. J. H. Lawton, Nature 355, 19 (1992)

9. J. C. Moore et al., Ecology 77, 694 (1996).

10. R. M. May, Stability and Complexity in Model Ecosystems (Princeton Univ. Press, Princeton, NJ, 1973).

11. Interaction strengths, partial derivatives of species' growth equations in equilibrium, can be expressed in Lotka-Voterra terms as $\alpha_{i j}=c_{i j} X_{i}^{*}$, where $c_{i j}$ is a coefficient of interaction between species $j$ and $i$, and $X_{i}^{*}$ is the equilibrium population size of species $i(10)$ We obtained $X_{i}^{*}$ from the observed biomass densities and calculated equilibrium feeding rates $F_{i j}{ }^{*}$ with equilibrium population sizes and published values of relative prey preferences, conversion efficiencies, and specific natural death rates $(15,16)$. By assuming $F_{i j}{ }^{*}$ $=c_{i j} X_{i}^{*} X_{j}^{*}$, we could then calculate interaction strengths between predator $j$ and prey $i(5): \alpha_{i j}=$ $-\left(F_{i j}^{*} / X_{j}^{*}\right)$ for the top-down effects (negative interaction strengths) and $\alpha_{j i}=e_{i j}\left(F_{i j}^{*} / X_{i}^{*}\right)$ for the bottomup effects (positive interaction strengths), where $e_{i j}$ is the efficiency with which food is converted into predator biomass. Note that top-down effects are per predator feeding rates and do not necessarily reflect the effects of removal of a predator on prey abundance, such as those established in manipulation experiments $(1,2)$. Intraspecific interaction strengths were assumed to be proportional to the natural death rates (15) according to $\alpha_{i i}=s \times d_{i}, i=1, \ldots, n(5)$, where $d_{i}$ refers to the natural specific death rate (per year) of species $\left(d_{i}<0\right)$ and $s$ (dimensionless) is the stability measure, the value leading to the minimum intraspecific interaction needed for matrix stability.

12. P. Yodzis, Introduction to Theoretical Ecology (Harper \& Row, New York, 1989).

13. R. Levins, in Mathematics and the Life Sciences, D. E. Matthews, Ed., vol. 18 of Lecture Notes in Biomathematics (Springer-Verlag, New York, 1977), pp. 153-199.

14. J. Hofbauer, K. Sigmund, The Theory of Evolution and Dynamical Systems (Cambridge Univ. Press, Cambridge, 1988), pp. 193, 204-206.
15. H. W. Hunt et al., Biol. Fertil. Soils 3, 57 (1987).

16. P. C. De Ruiter, J. A. Van Veen, J. C. Moore, L. Brussaard, H. W. Hunt, Plant Soil 157, 263 (1993).

17. P. C. De Ruiter, A. M. Neutel, J. C. Moore, Appl. Soil Ecol. 10, 217 (1998).

18. J. C. Moore, D. E. Walter, H. W. Hunt, Annu. Rev. Entomol. 33, 419 (1988)

19. The argument is as follows [for details see (22)]: A real $n \times n$ matrix, $\mathbf{A}=\left(\alpha_{i j}\right)$, is quasi-diagonally dominant (qdd) if there exists a set of $n$ positive numbers $\pi_{1}, \pi_{2}, \ldots, \pi_{n}$, such that the condition

$$
\pi_{i} \alpha_{i i}+\sum_{j \neq i}^{n} \pi_{j}\left|\alpha_{i j}\right|<0
$$

holds for every $i=1,2, \ldots, n$. If a community matrix is qdd, it is also stable $(14,28)$. Assume $\alpha_{i i}=\alpha<0$ for all $i$, and define a positive matrix $\underline{\mathbf{A}}$ with $\alpha_{i j}=\left|\alpha_{i j}\right|$ and $\alpha_{i i}=0$. Then the dominant eigenvalue $\lambda_{d}(\mathbf{A})$ of $\underline{A}$ is the smallest value for intraspecific interaction necessary for $\mathbf{A}$ to be qdd and hence sufficient for stability. A basic result of Krasnosel'skij et al. (29) then shows that this dominant eigenvalue $\lambda_{d}(\underline{\mathbf{A}})$ is larger than or equal to the maximum of all loop weights of $\mathbf{A}$, and hence of $\mathbf{A}$, because loop weights are defined in terms of absolute values of the matrix elements forming the loops

$$
\lambda_{d}(\underline{\mathbf{A}})=\lambda_{d}\left(\underline{\mathbf{A}}^{T}\right) \geq \max _{k, E_{k}}\left\{\left(\underline{\alpha_{i_{1} i_{2}} \alpha_{i_{2} i_{3}}} \cdots \underline{\alpha_{i_{k} j_{1}}}\right)^{1 / \mathrm{k}}\right\}
$$

where $E_{k}$ is the set of loops of length $k$. Thus, we used maximum loop weight as an approximation of the level of intraspecific interaction sufficient for stability.

20. We randomized matrices by exchanging the values of each predator-prey pair of interaction strengths $\alpha_{i j}=$ $-v, \alpha_{j i}=w$ with the values of a randomly chosen other predator-prey pair $\alpha_{k l}=-y, \alpha_{l k}=z$, becoming $\alpha_{i j}=-y, \alpha_{j i}=z$ and $\alpha_{k l}=-v, \alpha_{l k}=w$, where $j$ and $l$ are predators, $i$ and $k$ are prey, and $v, w, y$, and $z$ are positive numbers (7). The randomization preserved the sign structure representing the qualitative foodweb relations and the pairing of predator-prey values, but the biologically plausible distribution of interaction strengths was lost.

21. S. L. Pimm, J. H. Lawton, Nature 268, 329 (1977).

22. Details of the sensitivity analysis, the procedure attributing biomass pyramids, and the mathematical argument are available on Science Online at www. sciencemag.org/cgi/content/full/296/5570/1120/ DC1.

23. In the observed food webs, species do not always feed according to prey abundance. But despite a relative preference for higher trophic-level prey of some omnivores, lower level prey still tend to form the larger part of their diets.

24. J. E. Cohen, F. Briand, C. M. Newman, Community Food Webs: Data and Theory (Springer-Verlag, Berlin, 1990).

25. S. J. Hall, D. G. Raffaelli, in Advances in Ecological Research, M. Begon, A. H. Fitter, Eds. (Academic Press, London, 1993), pp. 187-239.

26. E. P. Odum, Fundamentals of Ecology (Saunders, Philadelphia, PA, ed. 3, 1971).

27. The results did not depend on exact population sizes as we measured them, but rather on differences in orders of magnitude of these population sizes. The results also did not simply follow from the equilibrium assumptions underlying the calculations, because using random input values for population sizes instead of observed values led to a large decrease in stability (5).

28. D. O. Logofet, Matrices and Graphs: Stability Problems in Mathematical Ecology (CRC Press, Boca Raton, FL, 1993), pp. 128, 156.

29. J. Krasnosel'skij, A. Lifshits, A. V. Sobolev, Positive Linear Systems (Heldermann, Berlin, 1989), pp. 169-179.

30. We thank P. Hogeweg, W. H. van der Putten, E. A. Neutel, J. Harvey, H. Olff, D. G. Raffaelli, C. Moore, and an anonymous referee for valuable comments on our work and earlier manuscripts, and C. Kaldeway for carrying out calculations on the 104 food webs from Cohen et al. (24).

26 November 2001; accepted 27 February 2002 
post date 23 August 2002

REPORTS: "Stability in real food webs: weak links in long loops" by A.-M. Neutel et al. (10 May 2002, p. 1120). A minus sign was missing from an equation on lines 10 and 11 of reference 11 . It should read " $F_{i j}^{*}=-c_{i j} X_{i}^{*} X_{j}^{*}$." 